\title{
CIÊNCIAPNATURA
}

\section{Uma análise da sazonalidade dos fluxos superficiais de energia e carbono em um ecossistema de campos nativos inserido no bioma Pampa}

An analysis of the seasonality of surface energy and carbon fluxes in an ecosystem of native fields inserted in the Pampa biome

\author{
Ricardo Acosta ${ }^{1}$, Debora Roberti ${ }^{1}$, Gisele Rubert ${ }^{1}$, Marcelo Diaz ${ }^{1}$, Ivan Cely ${ }^{1}$ e Osvaldo Moraes ${ }^{1,2}$ \\ ${ }^{1}$ Universidade Federal de Santa Maria, Santa Maria-RS, Brasil \\ ricardo.acosta@gmail.com, debora@ufsm.br, girubert@gmail.com, marbdiaz@gmail.com, mauriciocelytoro@gmail.com \\ ${ }^{2}$ Centro Nacional de Monitoramento e Alertas de Desastres Naturais, São José dos Campos-SP, Brasil \\ osvaldo.moraes@gmail.com
}

\begin{abstract}
Resumo
Neste trabalho, a técnica de covariância turbulenta foi utilizada para estimar os fluxos superficiais de carbono $\left(\mathrm{CO}_{2}\right)$ e de energia, na forma latente (LE) e sensivel (H), sobre um ecossistema de campo nativo (pastagem). Os resultados obtidos mostram que o ecossistema em questão apresenta uma predominante sazonalidade em seu funcionamento ecológico, em especial no que se refere as observações do fluxo de $\mathrm{LE}_{\text {e }} \mathrm{CO}_{2}$, com assimilação de carbono e evapotranspiração com valores máximos durante o verão. Durante a estação invernal, as observações apontam para um estado metabólico menor do ecossistema, com a assimilação de carbono quase nula (até mesmo uma inversão de sinal - fonte de carbono) e a taxa de evapotranspiração caindo a $60 \%$ de seu valor no estado de maior atividade metabólica.
\end{abstract}

Palavras-chave: Fluxo de energia, Fluxo de $\mathrm{CO}_{2}$, Bioma Pampa

\section{Abstract}

In this study, the eddy-covariance technique was used to estimate the surface fluxes of carbon (CO2) and energy, in the latent (LE) and sensible (H) forms, over a native field ecosystem (pasture). The results show that the ecosystem in question presents a predominant seasonality in its ecological functioning, especially regarding the observations of the $\mathrm{LE}$ and $\mathrm{CO} 2$ fluxes, with carbon assimilation and evapotranspiration with maximum values during the summer. During the winter season observations point to a lower metabolic state of the ecosystem, with almost zero carbon assimilation (even a carbon source signal inversion) and the evapotranspiration rate falling to $60 \%$ of its value in the highest state metabolic activity.

Keywords: Energy fluxes, $\mathrm{CO}_{2}$ fluxes, Pampa biome 


\section{Introdução}

O dióxido de carbono $\left(\mathrm{CO}_{2}\right)$ foi reconhecido como o principal gás efeito estufa responsável pelo aquecimento médio global do planeta relacionado às mudanças climáticas. O Painel Intergovernamental para as Mudanças Climáticas relatou um aumento significante de $40 \%$ na concentração atmosférica global de $\mathrm{CO}_{2}$ do valor pré-industrial, de aproximadamente 278 ppm em 1750 para 390,5 ppm em 2011. A taxa de crescimento anual de 2 ppm/ano na última década é reportada como maior que qualquer taxa decadal mediada anteriormente (STOCKER et al., 2013).

A preocupação com as mudanças no clima, cada vez mais evidente, aumentou o interesse em compreender os potenciais dos ecossistemas terrestres para reduzir o aumento dos níveis de $\mathrm{CO}_{2}$. Além disso, a quantificação dos estoques de carbono terrestre e a compreensão da variação temporal e espacial na absorção de carbono podem ajudar no desenvolvimento de estratégias de mitigação para o aumento do dióxido de carbono atmosférico. Os ecossistemas terrestres desempenham um papel crucial na dinâmica do carbono, trocando gases traços entre a atmosfera e a biosfera (MAGNANI et al., 2007). A produção primária bruta (GPP) dos ecossistemas terrestres é o maior fluxo global de $\mathrm{CO}_{2}$ com estimativas de $123 \pm 8$ Pg.C.ano ${ }^{-1}$ (BEER et al., 2010).

Dentre os inúmeros ecossistemas terrestres estudados, sabe-se, por conta de sua grande extensão na biosfera terrestre, que os ecossistemas de campo tem notável relevância no balanço global de carbono. Todavia, as informações são limitadas sobre a capacidade de assimilação de carbono em campos/pastagens. Na América do Sul, onde o ecossistema de campos nativos está estabelecido espacialmente e continuamente em três países (Brasil, Uruguai e Argentina), poucos estudos foram realizados. Uma vez que as investigações, até agora, são principalmente por curtos períodos de tempo, pontuais e com métodos simplificados, baseados em medidas de biomassa e análise de amostras de solo (LIMA et al., 2012), a capacidade de assimilação de $\mathrm{CO}_{2}$ deste complexo ecossistema permanece desconhecida. $\mathrm{O}$ presente estudo, através da aplicação do robusto método eddy covariance, fornece as primeiras medidas contínuas e precisas, em uma base de mais de 3 (três) anos de dados, das trocas de energia e carbono do ecossistema de campo nativo inseridos no bioma Pampa, região que se estende desde o sul do Brasil até centro-norte da Argentina.

\section{Materiais e métodos}

\subsection{Descrição do sítio, clima, solo e espécies de gramíneas}

Uma estação micrometeorológica, com medidas de alta frequência $(10 \mathrm{~Hz})$, com o sistema eddy covariance (EC) foi estabelecida em novembro de 2013 e, desde então, vem medindo continuamente as trocas entre a superfície-atmosfera. A atual torre de fluxo é uma das únicas estabelecidas no ecossistema de campos nativos do bioma Pampa. O sítio experimental (SM), latitude: 2943'27,502'’S e longitude: 5345’36,097' 'W, 88m de altitude, localiza-se no município de Santa Maria - RS, em uma área de 24 ha de campos nativos no Bioma Pampa e faz parte da rede PELD-CNPq (Programa de Pesquisa Ecológica de Longa Duração), sob supervisão da UFSM. O solo é classificado como Planossolo Háplico Eutrófico, segundo o mapa exploratório de solos do estado do RS (IBGE, 2002). Segundo a classificação de Köppen (NIMER, 1989), o clima pertence a tipificação “Cfa”, caracterizado por ter clima temperado úmido com verão quente. A vegetação encontrada na área de estudo, utilizada como pasto para o gado de corte, é de pastagem natural com predominância das seguintes espécies Andropogon lateralis, Axonopus affinis, Paspalum notatum, e Aristida laevis (LIPINSKI e SANTOS, 2014). Esta composição é distribuída uniformemente na área de estudo (QUADROS e PILAR, 2001). 
A coleta de dados iniciou-se em novembro de 2013 e permanece medindo continuamente. O sistema EC, conta com um anemômetro sônico 3-D Sonic Anemometer/WindMaster, da Gill Instruments e um analisador de gás infravermelho de caminho aberto LI-7200 Open Path $\mathrm{CO}_{2} / \mathrm{H}_{2} \mathrm{O}$ Analyser, da LI-COR para as medidas da densidade molar de vapor d'água e $\mathrm{CO}_{2}$, na altura de 3 (três) metros. Além do sistema $\mathrm{EC}$, está instalada uma estação meteorológica mensurando as principiais variáveis.

\subsection{Processamento dos dados}

O fluxo de $\mathrm{CO}_{2}$ (NEE) e os fluxos de energia, latente (LE) e sensível (H), necessários para o estudo foram obtidos por meio da técnica de covariância dos vórtices. Esta técnica baseia-se na covariância da velocidade vertical do vento e de um escalar obtidos dos dados experimentais em alta frequência pelo anemômetro sônico e o analisador de gás respectivamente (AUBINET et al., 2012). A técnica foi aplicada aos dados experimentais por meio do software EddyPro Advanced v6 (LI-COR version), resultando em fluxos médios em frequência de meia hora. A configuração do software adotada foi: i) cálculo das flutuações turbulentas em média por bloco; ii) rotação dupla; iii) correção para os efeitos da densidade; iv) a correção de atenuações de fluxo de acordo com e, finalmente; v) correções espectrais de alta e baixa frequência seguiram a metodologia de Moncrieff et al. (2004). Após o processamento dos fluxos, estes foram submetidos a um controle de qualidade para retiradas de dados espúrios seguindo indicações de Foken et al. (2012). Filtraram-se valores de fluxos superiores a $15 \mu \mathrm{molCO}{ }_{2} \cdot \mathrm{m}^{-2} \mathrm{~s}^{-1}, 350 \mathrm{~W} \cdot \mathrm{m}^{-2}$ e $600 \mathrm{~W} \cdot \mathrm{m}^{-2} \mathrm{e}$ inferiores a $-50 \mu \mathrm{molCO}_{2} \cdot \mathrm{m}^{-2} \mathrm{~s}^{-}$ ${ }^{1},-150 \mathrm{~W} \cdot \mathrm{m}^{-2} \mathrm{e}-60 \mathrm{~W} \cdot \mathrm{m}^{-2}$ para NEE, H e LE, respectivamente. As medidas fora destes limiares são devidos a erros aleatórios no sistema de medidas e, portanto, não apresentam significado físico. As lacunas geradas pelos filtros e o mau funcionamento dos sensores na série temporal dos fluxos foi preenchida utilizando a técnica de marginal distribution sample seguindo o procedimento sugerido por Reichstein et al. (2005).

\section{Resultados e discussões}

Desde novembro de 2013, vem sendo medindo continuamente as componentes do fluxo de energia, a fim de uma melhor compreensão das trocas sobre o ecossistema de campo nativo. Observa-se a partir da Figura 1 os ciclos diários médios para cada mês observado. É evidente a sazonalidade notavelmente marcante no fluxo de calor latente, com valores máximos na estação de verão, com valor máximo de $300 \mathrm{~W} \cdot \mathrm{m}^{-2}$ no mês de janeiro. Este valor diminui para $105,4 \mathrm{~W} \cdot \mathrm{m}^{-2}$ no mês de junho, início do inverno, sendo assim uma variação de aproximadamente de $64 \%$.

No que diz respeito a variabilidade no fluxo de calor sensível não se nota acentuada variação, todavia é importante observar que no período invernal a uma inversão de como a energia é particionada sobre o ecossistema de campo nativo, sendo a maior parte da energia utilizada para regular a temperatura do ar sobre o ecossistema. Os resultados aqui mostrados corroboram com os valores do fluxo de $\mathrm{CO}_{2}$ (Figura 2) que mostra de maneira mais clara a taxa metabólica do ecossistema como um todo.

A Figura 2 mostra as observações do fluxo de $\mathrm{CO}_{2}$. Na Figura 2 (a), observa-se a série temporal das médias diárias de NEE (Net Ecosystem Exchange), acrescida de uma linha azul representando a média móvel com nível de confiança de 95\%. A sazonalidade é notadamente observada em todos os anos, mas destaca-se o primeiro período quente, entre janeiro e março de 2014, onde a assimilação de carbono pelo ecossistema foi menor que para o mesmo período dos outros anos. Isso deve-se a uma situação de estresse hídrico ocorrido por conta de persistentes altos valores de VPD (Vapour Pressure Deficit) na região (não mostrado), que fez com que as taxas de evapotranspiração aumentassem e, portanto, como mecanismos de defesa da vegetação houve um fechamento estomatal. Situação semelhante ocorreu abril de 2016. 


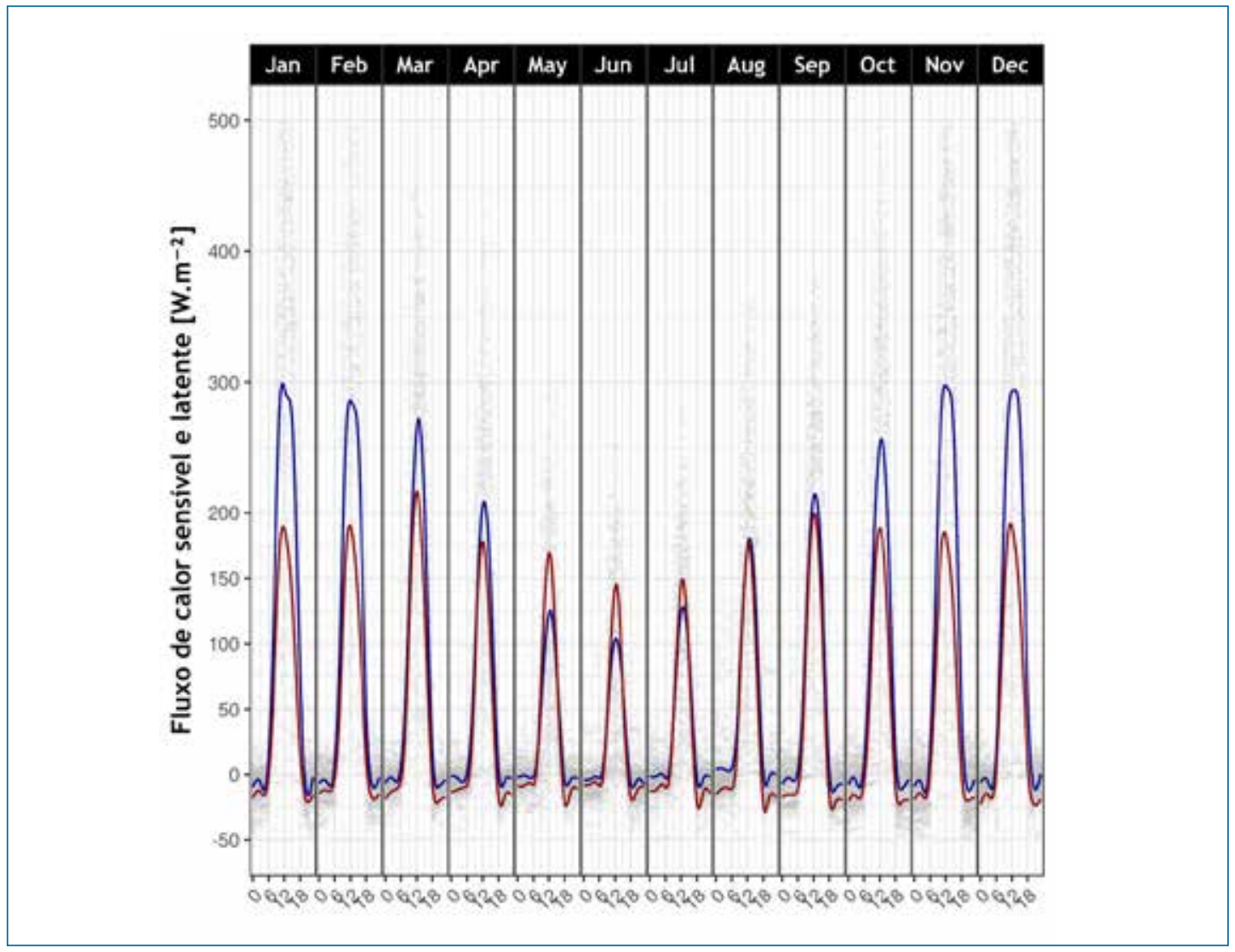

Figura 1 - Ciclo diário médio mensal para cada mês do período coletado de fluxos de calor sensível (linha vermelha) e latente (linha azul). As médias a cada meia-hora são mostrada com os pontos cinzas.

A Figura 2 (b), mostra a grande variabilidade nas taxas de fluxo de $\mathrm{CO}_{2}$ ao longo do ano, o que o difere de outros ecossistemas de campos localizados em sub-tropicais, outra vez verifica-se a sazonalidade, mas ressalta-se a mesma sazonalidade também atuando sobre a respiração do ecossistema (valores positivos de NEE).

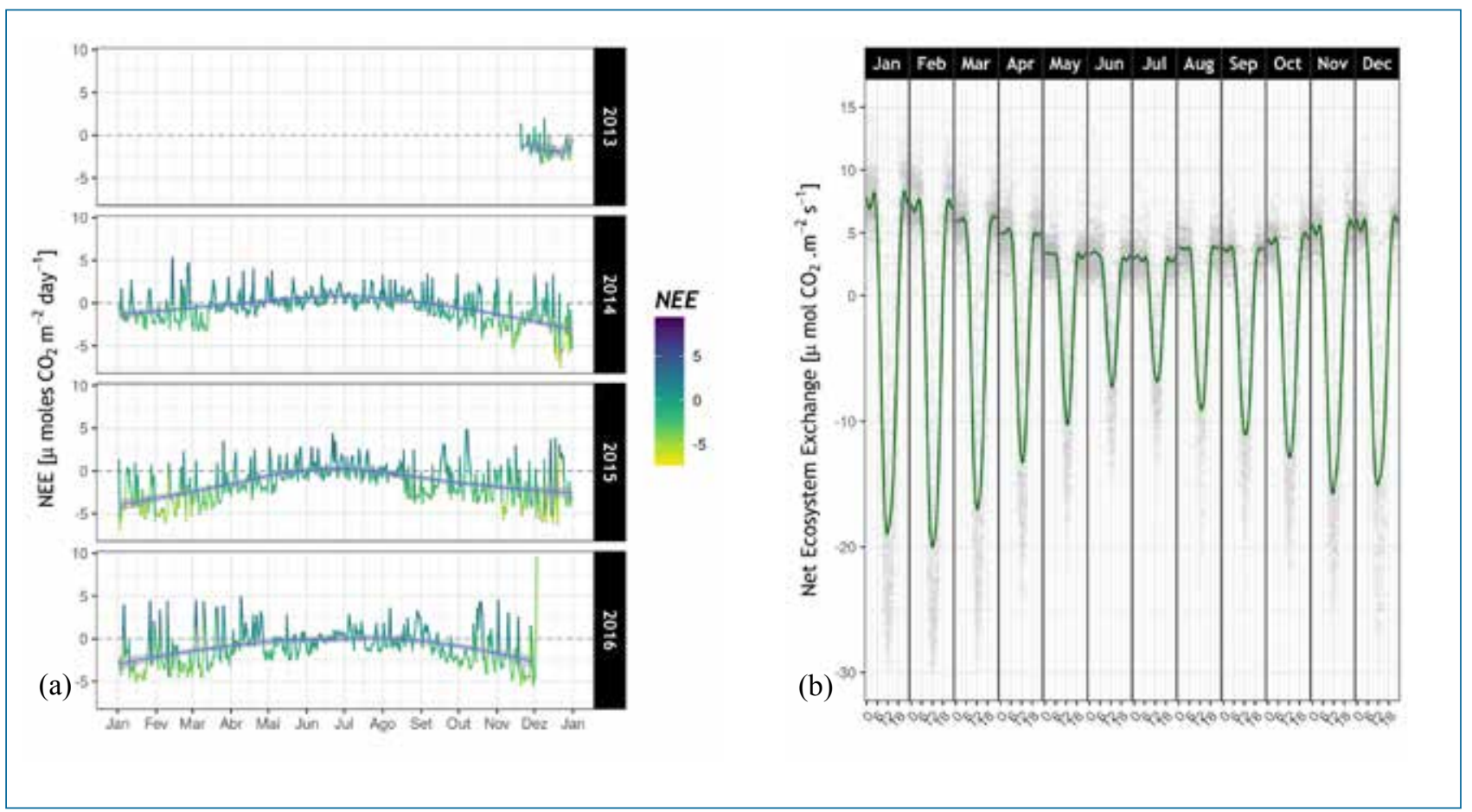

Figura 2 - (a) Série temporal do período coletado de NEE $\left(\mathrm{CO}_{2}\right)$ médio diário com linha da média com intervalo de confiança de 95\%; (b) Ciclo diário médio mensal, para cada mês do período coletado dos fluxos de $\mathrm{CO}_{2}$. As médias a cada meia-hora são mostradas com os pontos cinzas. 
A Figura 2b, mostra a grande variabilidade nas taxas de fluxo de $\mathrm{CO}_{2}$ ao longo do ano, o que o difere de outros ecossistemas de campos localizados em sub-tropicais, outra vez verifica-se a sazonalidade, mas ressalta-se a mesma sazonalidade também atuando sobre a respiração do ecossistemas (valores positivos de NEE).

\section{Conclusões}

O ecossistema de campo nativo no bioma Pampa apresenta alta variabilidade em seu funcionamento ecológico, notadamente evidenciado pela variação nos fluxos de água (calor latente) e fluxo de carbono. Na estação quente, o ecossistema está completamente ativo, com trocas substanciais entre o sistema solo-planta-atmosfera. Na estação invernal, a atividade metabólica do ecossistema diminui bastante. Ressalta-se o mecanismo de controle estomatal para situações de estresse hídrico causado pela atmosfera mais seca. Estes resultados indicam a importância de estudar os ecossistemas inseridos no bioma Pampa pelo relevante papel de seus serviços ambientais em área tão extensa onde está situado e, além disso, na interação superfície-atmosfera no microclima da região.

\section{Referências}

AUBINET M, VESALA T, PAPALE D. In: Eddy Covariance. Dordrecht: Springer, 2012.

BEER C, REICHSTEIN M, TOMELLERI E, CIAIS P, JUNG M, CARVALHAIS N, et al. Terrestrial gross carbon dioxide uptake: global distribution and covariation with climate. Science. 2010;329(5993):834-8.

FOKEN T, LEUNING R, ONCLEY SR, MAUDER M, AUBINET M. Corrections and Data Quality Control In: AUBINET M, VESALA T, PAPALE D, editors. Eddy Covariance. Dordrecht: Springer; 2012. p. 85-131.

IBGE. Manual Técnico da Vegetação Brasileira. Instituto Brasileiro de Geografia e Estatística - IBGE, 2012. ISSN 0101-4234

LIMA MA, BODDEY R, RODRIGUES B, MACHADO P, URQUIAGA S. Estoques de carbono e emissões de gases de efeito estufa na agropecuária brasileira. Brasília: Embrapa, 2012.

LIPINSKIL V, SANTOS T. Structure and spatial organization of two anuran communities of the Pampa biome. Iheringia Série Zoologia. 2014; 104(4).

MAGNANI F, MENCUCCINI M, BORGHETTI M, BERBIGIER P, BERNINGER F, DELZON S, et al. The human footprint in the carbon cycle of temperate and boreal forests. Nature. 2007;447:849-851.

MONCRIEFF J, CLEMENT R, FINNIGAN J, MEYERS T. Averaging, Detrending, and Filtering of Eddy Covariance Time Series. In: LEE X, MASSMAN WJ, LAW BE, editors. Handbook of Micrometeorology: a guide for Surface Flux Measurements. Dordrecht: Kluwer Academic Publishers; 2004. p. 181-208.

NIMER E. Climatologia do Brasil. Rio de Janeiro: IBGE; 1989.

QUADROS FLF, PILLAR VP. Dinâmica vegetacional em pastagem natural submetida a tratamentos de queima e pastejo. Ciência Rural. 2001; 31: 863-8.

REICHSTEIN M, FALGE E, BALDOCCHI D, PAPALE D, AUBINET M, BERBIGIER P, et al. On the separation of net ecosystem exchange into assimilation and ecosystem respiration: review and improved algorithm. Global Change Biology. 2005;11:1424-1439.

STOCKER T, QIN D, PLATTNER G, ALEXANDER L, ALLEN S, BINDOFF N, et al. Technical summary. In: Climate Change 2013: The Physical Science Basis. Contribution of Working Group I to the Fifth Assessment Report of the Intergovernmental Panel on Climate Change. Cambridge University Press; 2013. p. 33-115. 
Debora Roberti1

Universidade Federal de Santa Maria, Santa Maria-RS, Brasil E-mail: debora@ufsm.br

Gisele Rubert

Universidade Federal de Santa Maria, Santa Maria-RS, Brasil E-mail: girubert@gmail.com

Marcelo Diaz

Universidade Federal de Santa Maria, Santa Maria-RS, Brasil E-mail:marbdiaz@gmail.com

Ivan Cely

Universidade Federal de Santa Maria, Santa Maria-RS, Brasil

E-mail: mauriciocelytoro@gmail.com

Osvaldo Moraes

Universidade Federal de Santa Maria, Santa Maria-RS, Brasil Centro Nacional de Monitoramento e Alertas de Desastres Naturais, São José dos Campos-SP, Brasil

E-mail: osvaldo.moraes@gmail.com 